\title{
Suspected neospora-associated myocarditis in a dog
}

\author{
Carlos F. Agudelo ${ }^{1}$, Jana Petrášová ${ }^{2}$, Petra Honajzerová ${ }^{1}$ \\ University of Veterinary and Pharmaceutical Sciences Brno, Faculty of Veterinary Medicine, \\ ${ }^{1}$ Small Animal Clinic, ${ }^{2}$ Department of Physiology, Brno, Czech Republic
}

Received February 18, 2015

Accepted December 8, 2015

\begin{abstract}
Neosporosis was diagnosed in an American Staffordshire Terrier with collapse and arrhythmia episodes by serological and cardiovascular examination. Clinical signs, diagnosis and treatment of Neospora caninum are described. The dog did not develop any neurological abnormality, which is the most common manifestation. Furthermore, the dog was also serologically positive for antibodies to Toxoplasma gondii and negative for Bartonella henselae and Borrelia burgdorferi. Serological diagnosis was achieved by indirect immunofluorescence. The arrhythmia, cardiac markers, biochemistry, complete blood count and clinical signs gradually improved following therapy with clindamycin and antiarrhythmic therapy. After 3 months of therapy Toxoplasma gondii titres persisted low but Neospora caninum titres changed to negative values. According to the veterinary literature and to the authors' knowledge this was one of the few cases successfully treated for this condition. These observations demonstrated that neosporosis should be considered in the differential diagnosis of myocarditis in dogs and can be successfully treated by supportive therapy and clindamycin.
\end{abstract}

Infectious diseases, canine, cardiology, cardiac markers, serology

Neosporosis is a parasitic disease caused by the coccidian parasite Neospora caninum, which was first described no longer than 30 years ago (Dubey 1999). It has a worldwide distribution and affects dogs and other canides like coyotes, foxes, and wild dogs (Cringoli et al. 2002; Reichel et al. 2007; Lyon 2010), which are intermediate and definitive hosts of the parasite (Dubey et al. 1990; Tranas et al. 1999). Any breed or type of dog may be affected. Prevalence of infection in dogs varies from $0.5 \%$ to over 30\% (Cringoli et al. 2002; Reichel et al. 2007; Yagoob 2011). Transmission can occur vertically and horizontally through ingestion of infected tissues (Crookshanks et al. 2007). Neospora caninum also occurs in cattle (where it is an important cause of abortion), sheep, goats, deer and horses (Dubey et al. 1990; La Perle et al. 2001). Information on human neosporosis is scarce and it is presumed to be subclinical.

The most common clinical manifestations in dogs are peripheral neuromuscular deficits and central nervous system symptomatology like paresis/paralysis of forelimbs, ataxia, gait abnormalities, altered behaviour, blindness, head tilt, tremors, and seizures (Barber and Trees 1996). Abortions, collapse and sudden death also have been reported. Most of the cases of clinical disease are reported in dogs under 1 year of age (Dubey et al. 1990; Barber and Trees 1996), but can occur in older dogs, too (Lyon 2010). Neospora caninum also invades various tissues including the heart, lungs, gastrointestinal system, and skin, causing myocarditis, pneumonia, pancreatitis, hepatitis, adrenitis, and dermatitis (La Perle et al. 2001; Cringoli et al. 2002; Crookshanks et al. 2007; Reichel et al. 2007). Patients which are immunosuppressed are at a higher risk of developing clinical signs (Reichel et al. 2007).

Neosporosis can be diagnosed by serology using indirect fluorescent antibody test (IFAT) (Dubey et al. 1999; La Perle et al. 2001), direct agglutination or ELISA. Dogs are rarely 
reported as definitive hosts, which shed oocysts without developing a systemic infection and seroconversion (Cringoli et al. 2002; Meseck et al. 2005). Criteria used for serum samples to be considered positive are quite variable depending on the experience and the laboratory technique. Different dilutions have been considered as positive in different tests. Indirect fluorescent antibody test IFAT titres can range from 1:16 to 1:100 (Barber and Trees 1996). In general, a titre of 1:50 or more is considered positive evidence of exposure to $N$. caninum and could be considered supportive evidence of infection in patients with clinical symptoms (Dubey et al. 1990; Barber and Trees 1996).

Prevention can be done by limiting faecal contamination of livestock, avoiding ingestion of raw meats, restriction of unselective hunting and excluding infected dams that facilitate repeated transplacental infection from breeding (Crookshanks et al. 2007). Administration of glucocorticoids and other immunosuppressive drugs to seropositive dogs should be avoided as much as possible (La Perle et al. 2001; Lyon 2010). We describe a clinical case in a dog where clinical and laboratory findings were compatible with acute myocarditis, moreover the patient was positive to $N$. caninum showing seroconversion from positive titres to a negative value after appropriate therapy. To the authors' knowledge this could be the first case of successfully treated myocardial neosporosis.

\section{Case report}

A 12-year-old, female, castrated, $25 \mathrm{~kg}$, American Staffordshire terrier was presented to the Small Animal Clinic at the University of Veterinary and Pharmaceutical Sciences, Brno, Czech Republic, with a history of sudden collapse episodes. The dog suffered a fainting episode followed by respiratory arrest. After spontaneous return of vital signs the owner immediately sought medical care. The dog was currently on vaccination and deworming. On presentation, the dog was unable to stand; the mucous membranes were pale, auscultation revealed tachycardia $(240 \mathrm{bpm})$ with irregular and asynchronic pulses. No murmurs or gallop rhythms were detected. The respiratory rate was $60 \mathrm{rpm}$ with normal bronchovesicular sounds.

An electrocardiogram (ECG) revealed bursts of polymorphic ventricular arrhythmia (Plate XII, Fig. 1). Thoracic radiographs demonstrated a mild enlarged cardiac silhouette without pulmonary venous congestion and normal pulmonary patterns (Plate XII, Fig. 2). A complete blood count (CBC) revealed polycytaemia (erythrocytes 9.77 $10^{12} / 1$, reference range 5.5-8.5; haematocrit $68 \%$, reference range $35-55 \%$ ) and serum chemistries revealed increased activity of AST $(2.4 \mu \mathrm{kat} / 1$, reference range $0-1 \mu \mathrm{kat} / \mathrm{l})$ and ALT (2.42 $\mu \mathrm{kat} / \mathrm{l}$, reference range $0-1 \mu \mathrm{kat} / \mathrm{l})$. An abdominal ultrasound did not display any abnormalities.

Based on the physical exam finding of tachycardia and the ECG abnormalities the first stabilization was performed by using lidocaine $(2 \mathrm{mg} / \mathrm{kg}$ slow IV bolus, continued by CRI $50 \mu \mathrm{g} / \mathrm{kg} / \mathrm{min}$ ), oxygen therapy, infusion therapy (lactated Ringer's solution and glucose at $80 \mathrm{ml} / \mathrm{kg} /$ day) and cage rest. On the $2^{\text {nd }}$ day the dog was clinically bright and able to walk and drink. Another ECG demonstrated presence of ventricular premature complexes (VPCs) in more than $20 \%$ of the tracing. Echocardiography showed a mild regurgitation through the aortic and mitral valves (at the time of VPCs). Continual ECG examination was set during $24 \mathrm{~h}$ (Table 1). During continual ECG clinical signs of fainting or laboured breathing were not observed. According to the patient's clinical chart, on the same day she was examined several times and taken outside for urination and defecation. During that time several forms of supraventricular (SV) and ventricular arrhythmias were observed (Plate XII, Fig 3, Plate XIII, Figs 4, and 5). During the sleeping period the heart rate and the presence of arrhythmia were considerably reduced. The VPCs were monomorphic and probably originated from the left ventricle due to their negative morphology (Plate XII, Figs 2 and 3). Amiodarone ( $7 \mathrm{mg} / \mathrm{kg}$ PO, BID) was added to the therapy. 
Table 1. Results of continual ECG on 3 different days. Hospitalization (Day 1), a week after discharge (Day 13) and 3 months after therapy (Day 116).

\begin{tabular}{lrcc}
\hline ECG indicator & Day 1 & Day 13 & Day 116 \\
\hline Min. Rate & 42 & 55 & 44 \\
Max. Rate & 209 & 260 & 263 \\
Mean Rate & 78 & 68 & 73 \\
Supraventricular Ectopy & & & \\
Singles & 3307 & 46 & 0 \\
Couplets & 48 & 2 & 0 \\
Runs & 42 & 2 & 0 \\
Total & 3725 & 56 & 0 \\
Ventricular Ectopy & & & \\
Singles & 3699 & 211 & 92 \\
Couplets & 522 & 11 & 1 \\
Runs & 726 & 47 & 0 \\
R on T phenomena & 154 & 0 & 0 \\
Total & 10287 & 1009 & 101 \\
\hline
\end{tabular}

On the $3^{\text {rd }}$ day the dog's clinical status and the arrhythmia improved. Lidocaine therapy was discontinued. Systolic blood pressure measured with Doppler system was normal (150 $\mathrm{mm} \mathrm{Hg}$ ). The CBC and biochemistry indicatos returned to normal values. Based on the history (lack of evidence of myocardial trauma or ongoing heart disease) and ancillary tests, a tentative diagnosis of myocarditis was established. Troponin T $(0.065$ ug/l, range: < 0.01), and serology for antinuclear antibody (ANA) test (negative), Toxoplasma gondii (IgM negative, IgG: 1:40 positive), Neospora caninum (IgM 1:20, IgG 1:80), Bartonella henselae (IgM negative, IgG negative) and Borrelia burgdorferi (IgM: 0.27, IgG: 0.82; negative is considered when titre $\leq 0.84)$ were evaluated. On the $4^{\text {th }}$ the patient's ECG revealed sporadic presence of monomorphic VPCs but was clinically normal. Due to the serological activity of Neospora caninum clindamycin ( $24 \mathrm{mg} / \mathrm{kg}$ PO, BID) was added to the therapy. Amiodarone also was continued and the dog was discharged from the hospital.

The dog returned after 2 weeks for a follow-up. The owner reported that the dog was doing well, drinking and eating and receiving medication. Rest ECG showed sporadic ventricular bigeminismus. Another continual ECG was performed (Table 1). Complete blood count and biochemistry (including troponin T) were within normal values. The results of another continual ECG examination revealed a considerable reduction in VPCs (90.1\%) and SV complexes (98.6\%), which are considered as successful antiarrhythmic therapy (Table 1). Serology for $N$. caninum (IgM 1:10, IgG 1:40) showed also reduction in antibody reaction. The therapy with amiodarone was recommended for another month whereas clindamycin was continued until the next follow-up. Approximately 3 months after hospitalization the dog returned and was clinically normal. A resting ECG showed 1 single VPC and a continual ECG showed 92 VPCs a no SV complexes in one day (Table 1). Complete blood count and biochemistry were within normal ranges and had normal abdominal ultrasound. Troponin $\mathrm{T}$ was also within normal ranges. Serology for $N$. caninum was negative. Medication was withdrawn. During the evolution of the patient the antibodies IgG for $T$. gondii persisted on titre $\operatorname{IgG} 1: 40$. The patient returned after a year for traumatic lameness and otherwise was doing very well. No follow-ups were done after that.

\section{Discussion}

Most infectious myocarditis diagnosed in vivo has been determined based on antibody dynamics (Reichel et al. 2007; Lyon 2010). As we report, our patient developed serious episodes of arrhythmia (Figs 1, 3, 4, 5) leading to clinical signs of heart failure such as fainting. Since troponin T values at the beginning were elevated, a diagnosis of myocardial damage could be established. The most common causes of myocardial damage in dogs are structural changes due to overt heart disease, myocardial neoplastic infiltration 
and myocarditis. The last one includes trauma, nutritional deficiencies (carnitine or thiamine), toxic (doxorubicin), and immune-mediated causes (lupus erythematosus) in dogs (Dubey and Lappin 2011). Myocarditis is also reported in infections by bacteria (i. e. B. burgdorferi, B. henselae or Streptococcus spp.), fungi (cryptococcosis, coccidioidomycosis or aspergillosis), viruses (parvovirosis), rickettsia, and infestations by protozoa such as T. gondii, Trypanosoma cruzi, Sarcosporidia spp. or N. caninum.

Neospora caninum infects multiple tissues, predominantly CNS, peripheral nerves and muscles due to the affinity of the parasite to these tissues. One large report described progressive hind limb ataxia or paresis and signs of head and neck involvement (head tilt, ocular abnormalities and dysphagia) as the most common clinical signs in 27 cases with confirmed neosporosis. Interestingly, the same study presented also 2 dogs with sudden collapse due to cardiac manifestations of neosporosis (Barber and Trees 1996). A history of collapses in a patient with heart disease may be due to severely decreased cardiac output or arrhythmia. The last could be confirmed in the first clinical exam and ECG in our patient. Other signs in affected animals related with neosporal myocarditis are cyanosis, weakness and sudden death (Odin and Dubey 1993; Dubey et al. 2005). The scientific literature reports four cases of myocarditis associated with $N$. caninum in dogs: a 2-day-old Labrador Retriever (Barber and Trees 1996), a 4-week-old Flat-coated Retriever (Barber and Trees 1996), a 3-year-old male Mastiff dog (Odin and Dubey 1993) and a 10-monthold female Bull Mastiff (Thate and Laanen 1998). In the last two dogs the pathological description and previous decreased immunity associated condition (demodecosis) suggests a possible immune-mediated susceptibility to the parasite for the breed (Odin and Dubey 1993). Neosporal myocarditis was also reported in other species. A calf (Dubey et al. 1990) and a white rhinoceros (Williams et al. 2002) were confirmed lastly post mortem after sudden death. In general, myocardial lesions can develop when tachyzoites multiply rapidly within cells, causing rupture (Crookshanks et al. 2007). This is the rationale of the measurement of a specific cardiac marker such as troponins, which remains stable during a certain amount of time after sampling and is very specific for myocytes making them very useful for the diagnosis of myocardial damage.

The thoracic radiographs, echocardiography, ECG and blood work in our patient did not demonstrate any autoimmune processes, structural alterations or infiltration. However, serology was positive for $N$. caninum at values considered positive. As oocysts are rarely found in dog faeces, serological surveys provide the main clue for estimating the prevalence of canine neosporosis (Dubey et al. 1990). Diagnosis is most often presumptive based on patient history, clinical signs, ruling out other differential diagnosis and positive serology (IgM or IgG) using (IFAT), which is the standard for measuring serum antibodies against $N$. caninum (Dubey et al. 1999; Yagoob 2011), however IgM detection may not be rewarding, especially in congenitally infected dogs (Crookshanks et al. 2007). Dogs typically seroconvert two to three weeks after infestation with $N$. caninum thus the diagnosis of neosporosis in a live animal can be based on the combination of clinical signs with serology (Reichel et al. 2007). Titre magnitude does not correlate to the severity of clinical signs (Lyon 2010) and there is high evidence that a large portion of infection occurs in a subclinical form (Yagoob 2011). In general, a titre $\geq 1: 50$ of IgG are considered positive for exposure but as was indicated, in conjunction with clinical signs may be highly suggestive of infestation (Cringoli et al. 2002; Lyon 2010). Most dogs with clinical neosporosis have had antibody titres of at 1:200 (Mayhew et al. 1991; Crookshanks et al. 2007; Lyon 2010), although some may be seronegative at the time of testing. Some dogs that suffered from clinical neosporosis and that were confirmed by histological confirmation and isolation of the parasite showed IFAT titres of 1:50 of IgG or IgM. A high titre (i. e. 1:800) is often indicative of acute infection (Dubey et al. 1990; La Perle et al. 2001; Silvaa et al. 2007; Yagoob 2011). There is also a potential cross-reactivity with 
T. gondii and N. hughesi. Neospora hughesi is a closely related organism whose biological and pathophysiological behaviour is still uncertain (Lyon 2010). Toxoplasma gondii is a parasite, which structurally, genetically and immunologically is very closely related to $N$. caninum. Due to the these similarities between these two organisms, serological diagnosis should be aware of potential of false positive results due to antigenic cross reactivity (Silvaa et al. 2007; Dubey and Lappin 2011). Moreover, the negative paired control for T. gondii antibodies were unchanged, suggesting different dynamics in comparison with $N$. caninum. We can assume that the steady chronic titre of $T$. gondii and the reduction in the titre $N$. caninum is the result of an acute episode of $N$. caninum infection with rapid reaction to the therapy. The clinician should be aware that a single sample cannot differentiate between past and present exposure, and cannot on its own constitute a basis for diagnosis of an active infection. Simultaneous measurements of antibodies and paired-sample titres are recommended for detection of seroconversion (Crookshanks et al. 2007).

The source of infestation for this dog is uncertain, as she was fed brand dog food; she was not immunosuppressed and was not in contact with animal carcasses. Definitive diagnosis requires identification of organisms by biopsy samples and then using immunohistochemical staining (Crookshanks et al. 2007); however this was declined by the owner.

This is one of the few reported cases of neospore-associated myocarditis diagnosed and treated ante mortem in a dog. The untreated disease can course subclinically and some cases can result in death (La Perle et al. 2001; Lyon 2010); but the prognosis improves if therapy is started early (Lyon 2010). One large study described improvement of symptoms in 10 of 27 dogs with a total recovery reported only in 5 of them (Barber and Trees 1996). Other isolated reports described individual cases of muscular atrophy (Crookshanks et al. 2007), encephalomyelitis or polyradiculoneuritis (Mayhew et al. 1991). Particularly, all these cases manifested neurological signs; none of them dealing with cardiac manifestation. Current treatment protocols are largely extrapolated from recommendations for treatment of canine toxoplasmosis (Crookshanks et al. 2007; Lyon 2010). Clindamycin $(12.5-25 \mathrm{mg} / \mathrm{kg}$ PO or IM every $12 \mathrm{~h}$ for 4 weeks), trimethoprim sulphadiazine alone $(15-20 \mathrm{mg} / \mathrm{kg}$ PO every $12 \mathrm{~h}$ for 4 weeks) or in combination with pyrimethamine $(1 \mathrm{mg} / \mathrm{kg}$ PO every $24 \mathrm{~h}$ for 4 weeks) have been recommended for long periods or months (Cringoli et al. 2002; Dubey et al. 1990; Crookshanks et al. 2007; Dubey and Lappin 2011). Clindamycin affects multiplication of $N$. caninum tachyzoites but is thought to have little or no effect on bradyzoites (Dubey et al. 1990; Dubey and Lappin 2011). The rate of clindamycin absorption in dogs is rapid after oral administration and is comparable with absorption rates after intramuscular and subcutaneous injections (Lyon 2010). Corticosteroids have been shown to trigger or worsen clinical disease in dogs with neosporosis (La Perle et al. 2001), so they should not be administered. This case illustrates the potential for clinical improvement and remission following early diagnosis, treatment with antibiotics and supportive therapy of $N$. caninum infection.

\section{References}

Barber JS, Trees AJ. 1996: Clinical aspects of 27 cases of neosporosis in dogs. Vet Rec 139: 439-443

Cringoli G, Rinaldi L, Capuanob F, Baldib L, Venezianoa V, Capelli G 2002: Serological survey of Neospora caninum and Leishmania infantum co-infection in dogs. Vet Parasitol 106: 307-313

Crookshanks JL, Taylor SM, Haines DM, Shelton GD 2007: Treatment of canine pediatric Neospora caninum myositis following immunohistochemical identification of tachyzoites in muscle biopsies. Can Vet $\mathrm{J} \mathbf{4 8}$ : 506-508

Dubey JP 1999: Recent advances in Neospora and neosporosis. Vet Parasitol 84: 349-367

Dubey JP, Lappin M. 2011: Toxoplasmosis and neosporosis, 1376, p. 806-827. In: Greene CE (ed.): Infectious Diseases of the Dog and Cat, $4^{\text {th }}$ edn, Saunders

Dubey JP, Knickman E, Greene CE 2005: Neonatal Neospora caninum infections in dogs. Acta Parasitol 50: 176-179 
Dubey JP, Miller S, Lindsay DS, Topper MJ 1990: Neospora caninum-associated myocarditis and encephalitis in an aborted calf. J Vet Diagn Invest 2: 66-69

La Perle KMD, Del Piero F, Carr RF, Harris C, Stromberg PC 2001: Cutaneous neosporosis in two adult dogs on chronic immunosuppressive therapy. J Vet Diagn Invest 13: 252-255

Lyon C 2010: Update on the diagnosis and management of Neospora caninum infections in dogs. Top Companion Anim Med 25: 170-175

Mayhew IG, Smith KC, Dubey JP, Gatwards LK, McGlennon NJ 1991: Treatment of encephalomyelitis due to Neospora caninum in a litter of puppies. J Small Anim Pract 32: 609-612

Meseck EK, Njaa BL, Haley NJ, Park EH, Barr SC 2005: Use of a multiplex polymerase chain reaction to rapidly differentiate Neospora caninum from Toxoplasma gondii in an adult dog with necrotizing myocarditis and myocardial infarct. J Vet Diagn Invest 17: 565-568

Odin M, Dubey JP. 1993: Sudden death associated with Neospora caninum myocarditis in a dog. J Am Vet Med Assoc 203: 831-833

Reichel MP, Ellis JT, Dubey JP 2007: Neosporosis and hammondiosis in dogs. J Small Anim Pract 48: 308-312

Silvaa DA, Lobatoa J, Mineob THP, Mineoa JR 2007: Evaluation of serological tests for the-diagnosis of Neospora caninum infection in dogs: Optimization of cut off titers and inhibition studies of cross-reactivity with Toxoplasma gondii. Vet Parasitol 143: 234-244

Thate FM, Laanen SC. 1998: Successful treatment of neosporosis in an adult dog. Vet Q 1998: S113-S114

Tranas J, Heinzen RA, Weiss LM, McAllister MM 1999: Serological evidence of human infection with the protozoan Neospora caninum. Clin Diagn Lab Immunol 6: 765-767

Williams JH1, Espie I, van Wilpe E, Matthee A 2002: Neosporosis in a white rhinoceros (Ceratotherium simum) calf. J S Afr Vet Assoc 73: 38-43

Yagoob G. 2011: Seroprevalence of Neospora Caninum in Stray Dogs. Am J Anim Vet Sci 6: 100-104 
Plate XII

Agudelo C.F. et al.: Suspected ... pp. 85-90

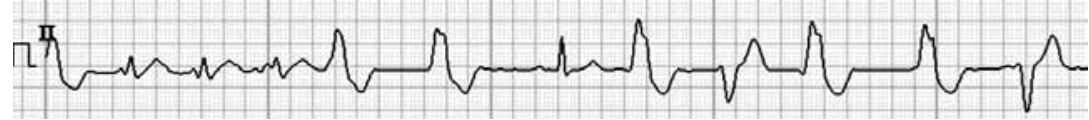

Fig. 1. Slow ventricular rhythm with polymorphic ventricular complexes. The $7^{\text {th }}$ complex from left to right is the only sinus complex in origin. The first 3 complexes from left to right are parasystoles. Lead II. Speed $50 \mathrm{~mm} / \mathrm{s}$, amplitude $5 \mathrm{~mm}=1 \mathrm{mV}$.

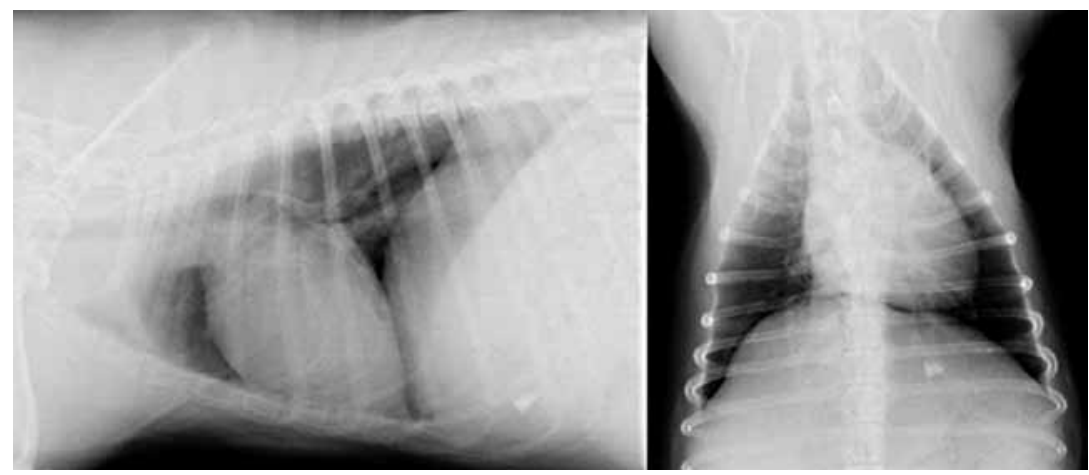

Fig. 2. Thoracic radiographs show a vertebral heart size of 11.2. Pulmonary lobes and vessels seem without abnormalities.

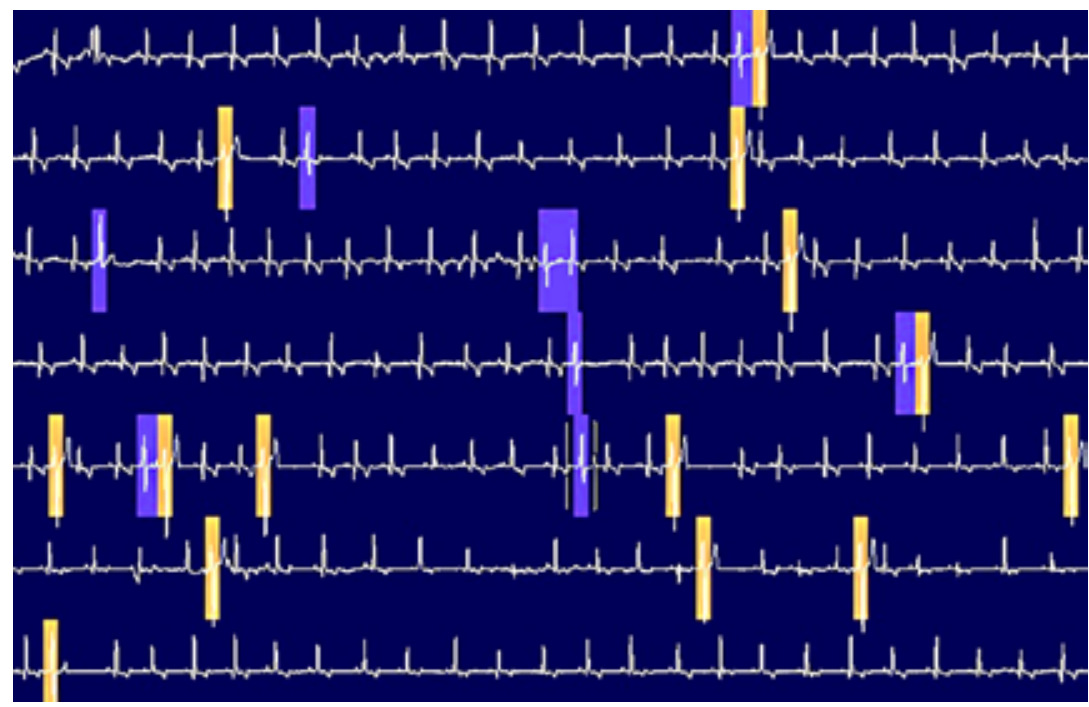

Fig. 3. Channel II continual ECG from the $2^{\text {nd }}$ day of hospitalization. Orange colour represents monomorphic isolated VPCs. Their negative morphology suggests left ventricular origin. In blue colour SV complexes are shown (P wave absence/change in morphology, mild changes in QRS-T morphology with compensatory pause). 


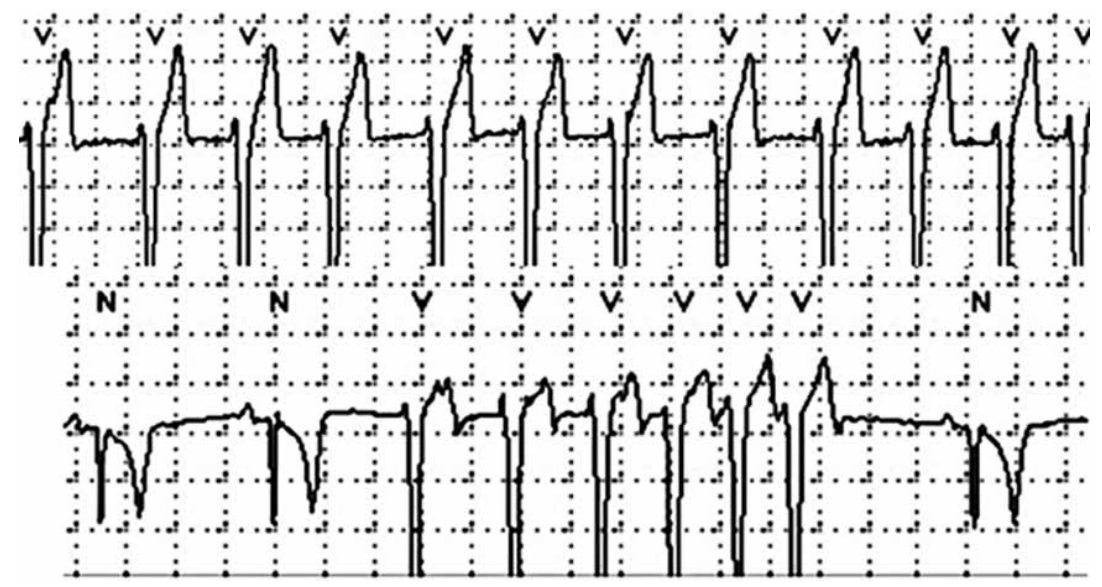

Fig. 4. The upper trace shows a long ventricular run with a rate of $130 \mathrm{bpm}$ (slow ventricular rhythm). The lower trace displays a burst of VPCs (left to right $3^{\text {rd }}$ to $6^{\text {th }}$ ) with a rate of 220 (ventricular tachycardia).

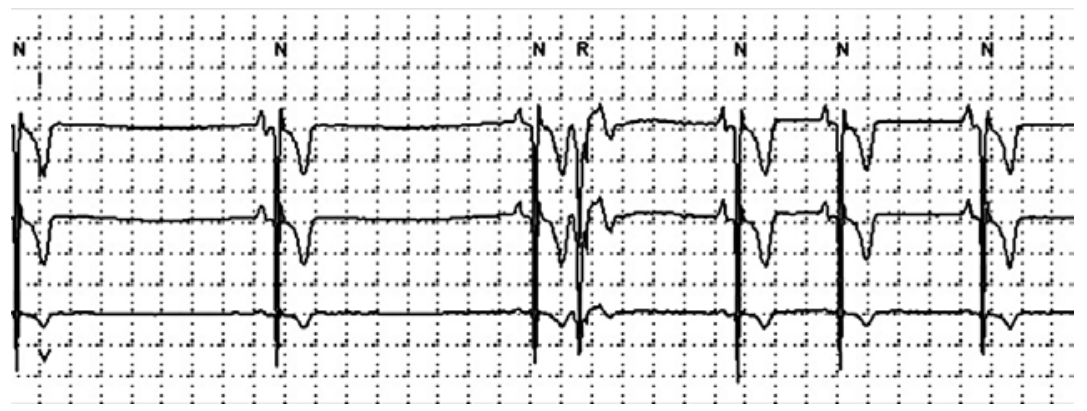

Fig. 5. $\mathrm{R}$ on $\mathrm{T}$ phenomenon (4 ${ }^{\text {th }} \mathrm{QRS}$ complex from left to right). The $\mathrm{R}$ wave of the $4^{\text {th }} \mathrm{VPC}$ is overimposed on the T wave of the preceding QRS complex. 